\title{
Conocimiento de las medidas de bioseguridad en personal de salud
}

Jorge A. Ruiz de Somocurcio Bertocchi ${ }^{1, a}$

\section{RESUMEN}

Objetivo: Determinar el nivel de conocimiento de las medidas de bioseguridad en el personal profesional del Hospital Nacional Hipólito Unanue (HNHU), cuantificar a los trabajadores por grupos con distintas características, y comparar analíticamente sus frecuencias y porcentajes, para reconocer a los grupos de mayor vulnerabilidad y focalizar futuras capacitaciones.

Materiales y métodos: En este estudio observacional, analítico y transversal, se incluyó a todo el personal de salud profesional del HNHU que haya aceptado resolver el cuestionario de evaluación. Se obtuvo una muestra de 567 trabajadores, con un margen de error aceptable en el 3.32\%, nivel de confianza del 99\% y usando un comportamiento de la población en el 50\%. Como instrumento de recolección de información, se utilizó una ficha tipo cuestionario con un total de 10 preguntas referentes a las medidas de bioseguridad, la cual fue entregada y repartida por la jefatura de cada servicio del HNHU. Se analizaron los datos según sus frecuencias, porcentajes y la prueba no paramétrica de chi cuadrado.

Resultados: El 21\% del personal evaluado obtuvo un resultado de 8 a 10 respuestas correctas, el 75\% de 4 a 7, y el $4 \%$ de 0 a 3. Existen diferencias significativas en el nivel de conocimiento según las variables de grupo ocupacional, edad, tiempo de trabajo en el hospital, sexo por grupo ocupacional, sexo por edad y el haber recibido inducción laboral.

Conclusiones: El conocimiento del personal profesional del hospital sobre las medidas de bioseguridad no es el ideal, lo cual genera una situación de alto riesgo biológico tanto para el personal profesional y técnico como para los pacientes. Es posible dar capacitaciones sobre bioseguridad focalizadas a los grupos más vulnerables, además de mejorar su calidad e impacto.

Palabras clave: Exposición a agentes biológicos; factores biológicos; personal de salud; conocimiento; medidas de seguridad; riesgos laborales; centros de salud; hospitales urbanos (Fuente: DeCS BIREME).

\section{Knowledge of biosecurity measures among health personnel}

\section{ABSTRACT}

Objective: To determine the level of knowledge of biosecurity measures among the professional personnel of the Hospital Nacional Hipólito Unanue (HNHU), to quantify the workers in groups by different features, and to analytically compare their frequencies and percentages, in order to recognize the most vulnerable groups and focus on them on future trainings.

Materials and methods: This observational, analytical and cross-sectional study included all professional health personnel of the HNHU who agreed to answer the assessment questionnaire. A sample of 567 workers was obtained, with an acceptable margin of error of 3.32\%, a confidence level of $99 \%$ and a population behavior of 50\%. A questionnairetype form with a total of 10 questions related to biosecurity measures was used as tool for collecting information, which was submitted and distributed by the head of each HNHU service. Data were analyzed according to their frequencies, percentages and the non-parametric chi square test.

Results: Twenty-one percent (21\%) of the evaluated personnel had a score of 8 to 10 correct answers, $75 \%$ of 4 to 7 , and $4 \%$ of 0 to 3 . There were significant differences in the level of knowledge based on variables, such as occupational group, age, tenure at the hospital, gender by occupational group, gender by age, and induction training.

Conclusions: Knowledge of biosecurity measures among the hospital's professional personnel is not the ideal one, leading to high biological risk for both the professional and technical personnel and the patients. It is possible to provide training on biosecurity focused on the most vulnerable groups, and to improve its quality and impact.

Keywords: Exposure to biological agents; biological factors; health personnel; knowledge; security measures; occupational risks; health centers; urban hospitals (Source: MeSH NLM).

1. Médico General.

a. Facultad de Medicina Humana, Universidad de San Martín de Porres. 


\section{INTRODUCCIÓN}

Las medidas de bioseguridad son el conjunto de conductas mínimas a ser adoptadas, a fin de reducir o eliminar los riesgos para el personal, la comunidad y el medio ambiente ${ }^{(1)}$. La bioseguridad en sí es un enfoque estratégico e integrado para el análisis y la gestión de los riesgos relativos a la vida y la salud ${ }^{(2)}$.

Cada labor o trabajo presenta distintos riesgos inminentes, llamado riesgo ocupacional, perjudicando tanto la actividad laboral como la economía y llevando al desarrollo de la medicina ocupacional. La OIT, informa en el año 2002, que al año mundialmente 270 millones de asalariados son víctimas de accidentes ocupacionales, y 160 millones contraen enfermedades profesionales ${ }^{(3)}$. El personal de salud tiene un mayor riesgo de lesiones ocupacionales que cualquier otro, por encontrarse en un ambiente laboral con una amplia gama de exposiciones nocivas ${ }^{(4)}$. Al estar expuestos a sangre y otros fluidos corporales durante su trabajo, corre el riesgo de sufrir infecciones virales (incluyendo los virus VHB, $\mathrm{VHC}$ y $\mathrm{VIH}$ ), bacterias, parásitos, toxinas u otros agentes patógenos, por distintas vías. Aquellos ubicados en las áreas de cirugía, cuartos de emergencia, central de equipos, recolección de desechos y laboratorios tienen mayor riesgo de exposición ${ }^{(5,6)}$. En el personal de salud, la proporción de la carga mundial de morbilidad atribuible a la exposición profesional es del $40 \%$ en caso de la Hepatitis B y C, y $2.5 \%$ en el caso del $\mathrm{VIH}$. El $90 \%$ de estas exposiciones suceden en países en vía de desarrollo ${ }^{(7)}$. En el HNHU se reportaron
103 accidentes ocupacionales durante el año 2011 (Fuente: Oficina de Epidemiología y Salud Ambiental). Las distintas estrategias para evitar los accidentes ocupacionales incluyen la implementación de las precauciones universales, la inmunización contra hepatitis $B$, garantizar equipos de protección personal y reporte del accidente con manejo de la post exposición ${ }^{(8)}$. Actualmente en el Perú se desconoce el nivel de eficacia del manejo de las medidas de bioseguridad en la mayoría de hospitales, además de desconocerse completamente el nivel de conocimiento del personal de salud sobre estas mismas medidas. El objetivo general de este estudio fue identificar y describir el nivel de conocimiento sobre las medidas de bioseguridad y analizar su asociación con las distintas características del personal profesional del HNHU. Estas variables fueron: el grupo ocupacional, el tiempo de trabajo en el HNHU, la edad, el sexo, el área de trabajo, el área de riesgo (Tabla 1), haber recibido inducción laboral al comenzar a trabajar para el hospital, haber sido capacitado sobre medidas de bioseguridad y la duración de dicha capacitación. Existiendo pocos estudios a nivel nacional sobre el tema y siendo ninguno semejante al presente, es de mi interés ofrecer una línea de base en cuanto al conocimiento sobre las medidas de bioseguridad del personal del HNHU.

Con los resultados de este estudio, será posible realizar capacitaciones dirigidas al personal del hospital con mayor vulnerabilidad. De esta forma se podrá, de una manera eficiente, mejorar la seguridad laboral y la calidad de atención en la institución.

Tabla 1. Áreas de trabajo por áreas de riesgo

\begin{tabular}{lll}
\multicolumn{1}{c}{ ALTO RIESGO } & MEDIANO RIESGO & BAJO RIESGO \\
Hemodiálisis & Neumología & Bien de Personal \\
Anestesiología & Pediatría & Farmacia \\
Emergencia & Diagnóstico por Imágenes & S.I.S. \\
Laboratorio & Rehabilitación & Nutrición \\
Cirugía Especialidades & Consultorios Externos & Servicio Social \\
Cirugía General & Epidemiologia y Salud & Jefatura de Enfermería \\
Cirugía de Tórax & Ambiental & \\
Gíneco-Obstetricia & \\
Neonatología & \\
Centro Quirúrgico & \\
Unidad de Terapia Intensiva & \\
Anatomía Patológica & \\
Recuperación & \\
Medicina & & \\
Esterilización & & \\
\end{tabular}




\section{MATERIALES Y MÉTODOS}

Se realizó un estudio de tipo analítico, prospectivo, transversal, observacional no experimental y de enfoque cualitativo. El universo trabajado fue de 908 trabajadores profesionales de la salud trabajando en el HNHU durante el segundo semestre del año 2011 y se buscó evaluar al universo completo.

La unidad de análisis fue el trabajador del HNHU, con criterios de inclusión que fueron el que sea profesional y que haya aceptado resolver el cuestionario, mientras que el criterio de exclusión fue que el profesional no esté relacionado al área de la salud.

Los datos se obtuvieron mediante un instrumento de recolección de datos de tipo cuestionario, el cual contiene una sección de filiación con las variables a analizar y otra sección de 10 preguntas relacionadas a las medidas de bioseguridad. Este fue diseñado en base a las normas generales de bioseguridad presentadas en el "Manual de Bioseguridad norma técnica N ${ }^{\circ} 15$ - MINSA / DGSP - V.01 (2004)"1. El instrumento de recolección de datos ha sido revisado por dos equipos de expertos. Un equipo de profesionales de la salud de la Dirección de Salud IV Lima Este y otro equipo de profesionales de la Oficina de Epidemiología y Salud Ambiental del HNHU.

Para calcular el margen de error aceptable y el nivel de confianza, bajo un comportamiento de la población (response distribution) de $50 \%$, se utilizó la función de
"Sample Size Calculator - Raosoft". De esta forma se obtuvo que de la muestra original de 567 fichas resueltas, se logró un margen de error aceptable de 3.32\%, un nivel de confianza del $99 \%$ con el comportamiento de la población de $50 \%{ }^{(9)}$.

Las variables consideradas, como grupo ocupacional, edad, tiempo de trabajo, etc., fueron relacionadas y analizadas con los grupos relativos al número de preguntas, relacionadas a las medidas de bioseguridad, contestadas correctamente. Estos grupos se dividieron en los siguientes: 8 a 10 respuestas correctas, 4 a 7 respuestas correctas y 0 a 3 respuestas correctas.

Se trabajaron las variables con una evaluación analítica en la que se consideraron frecuencias, porcentajes, medidas de tendencia central y pruebas no paramétricas como Chi cuadrado.

La posibilidad de la realización de este trabajo de investigación fue evaluada por el comité de ética del Hospital Nacional Hipólito Unanue y de la Facultad de Medicina de la Universidad San Martín de Porres. Se respetó la confidencialidad de la información del personal evaluado y participante del estudio.

\section{RESULTADOS}

De la muestra trabajada se obtuvo un $21 \%$ de profesionales con 8 a 10 respuestas correctas, $75 \%$ con 4 a 7 y $4 \%$ con 0 a 3 (Figura 1).

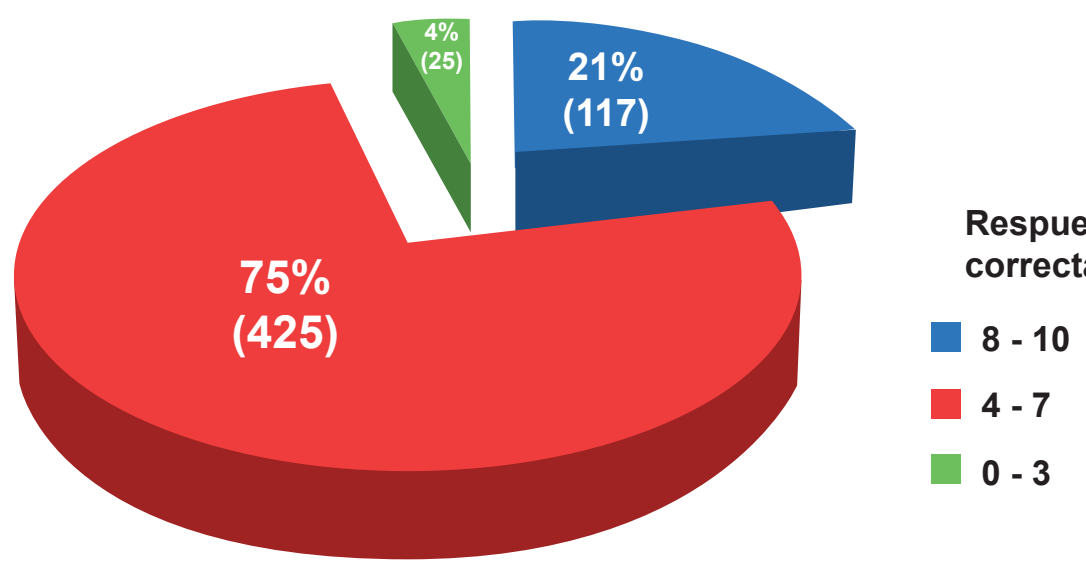

Figura 1. Nivel de conocimiento del personal profesional con porcentajes y frecuencias

De los trabajadores profesionales del HNHU el 71,5\% es femenino, mientras que aproximadamente el 28,5\% es masculino. Mas no se encontró una diferencia estadísticamente significativa del nivel de conocimiento sobre las medidas de bioseguridad entre ambos sexos. En relación a los años de experiencia, se encontró que el personal con más de 3 años de labor en el HNHU obtuvo resultados estadísticamente inferiores.
Al discernir por edad el grupo entre los 25 y 40 años de edad se obtuvo el resultado más alto, seguido de aquel entre los 25 y 60 años. Los grupos con resultados inferiores fueron aquellos menores de 25 años y mayores de 60 . El sexo femenino por edades presentó el mismo fenómeno que se vio al evaluar el personal en general por edades. Más al evaluar al sexo masculino, la diferencia por edades no fue significativa (Figura 2). 


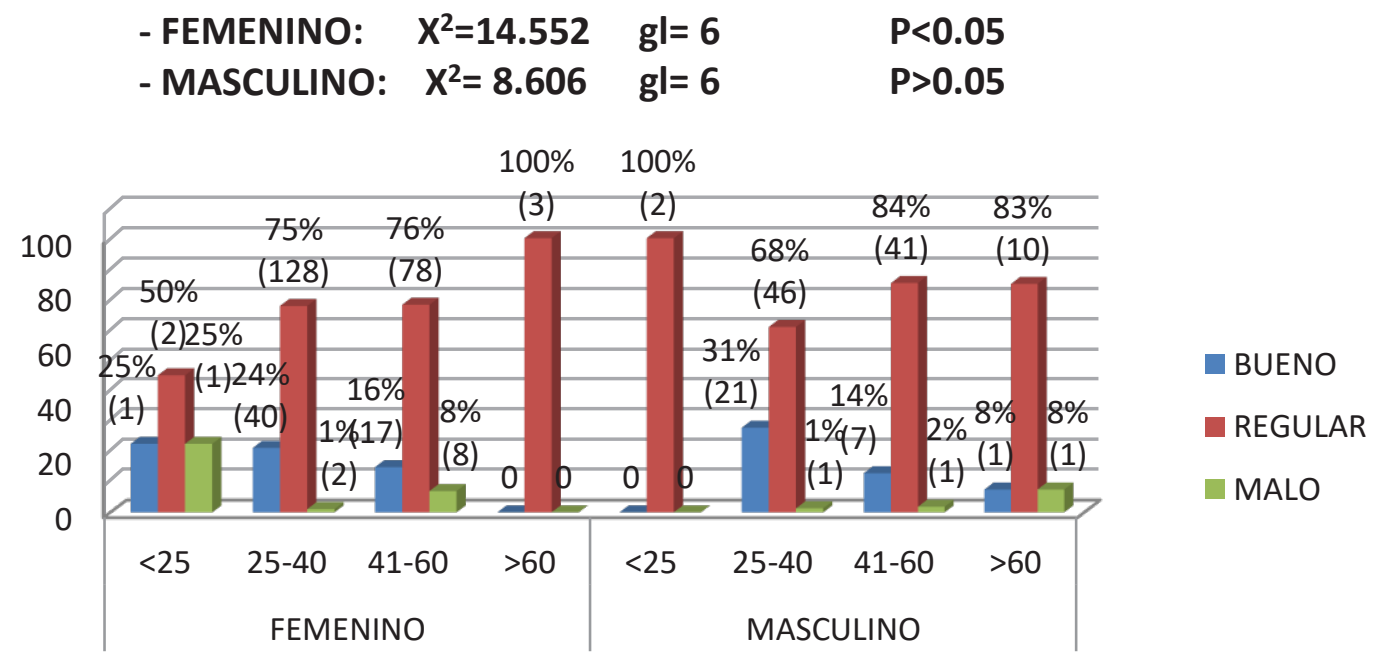

Figura 2. Análisis del nivel de conocimiento según sexo y edad con porcentajes y frecuencias

En cuanto a los grupos ocupacionales, se encontró que el mayor nivel de conocimiento es el personal médico. Seguido por los tecnólogos y obstetrices. El personal que presenta mayor vulnerabilidad es el de odontología, enfermería y nutrición. Al separar ambos sexos y compararlos por grupos ocupacionales, se halló una situación muy similar. En ambos sexos los grupos ocupacionales con mayor nivel de conocimiento fueron los del personal médico, seguidos por los tecnólogos y luego los enfermeros (Figura 3).

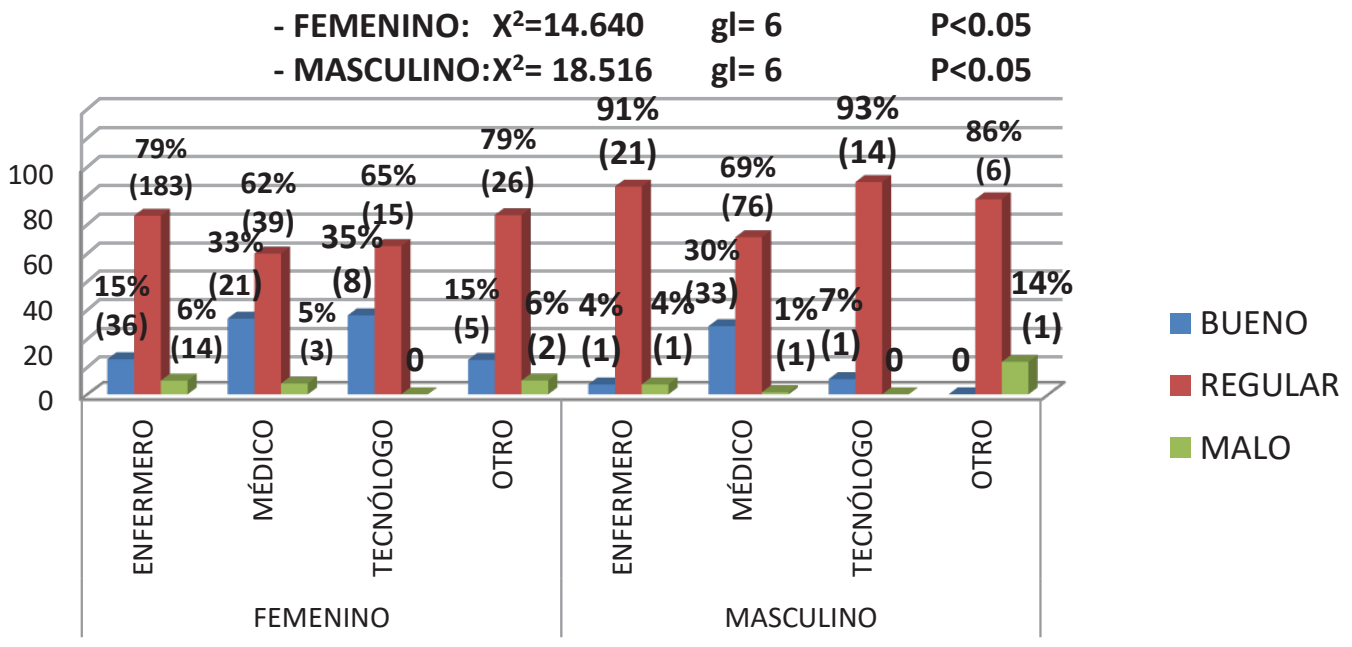

Figura 3. Nivel de conocimiento según sexo y grupo ocupacional con porcentajes y frecuencias

La diferencia de nivel de conocimiento entre las áreas de trabajo y de riesgo no fue estadísticamente significativa. En el estudio se obtuvo que el nivel de conocimiento entre el personal que afirma haber recibido una inducción laboral y el que lo niega es estadísticamente distinto, presentando aquellos que no recibieron inducción laboral paradójicamente un nivel de conocimiento superior.

No se halló diferencia del nivel de conocimiento, entre el personal que refiere haber recibido algún tipo de capacitación sobre bioseguridad y los que lo negaron. Así como tampoco lo hubo según la duración de la capacitación recibida.

De las diez preguntas del instrumento de recolección relacionadas al conocimiento sobre medidas de bioseguridad, aquellas referentes al proceder del personal posterior a la exposición a materiales potencialmente contaminados y al manejo y transporte de las muestras de laboratorio tomadas, fueron las que obtuvieron una menor frecuencia de respuestas correctas. 


\section{DISCUSIÓN}

Al comparar los resultados del nivel de conocimiento sobre medidas de bioseguridad en el personal del HNHU con estudios similares, encontramos que este tiende a ser inferior o semejante al de otros hospitales ${ }^{(10-13)}$. Uno de los estudios presentados coincide con el hallazgo de un mayor nivel de conocimiento por parte del personal médico sobre el de enfermería. Este mismo coincide con un nivel de conocimiento semejante en ambos sexos ${ }^{(13)}$. Resultados del estudio antes mencionado, y otro agregado, coincidieron en un menor nivel de conocimiento sobre bioseguridad relacionado a mayor edad y mayor tiempo laboral en una institución ${ }^{(13,14)}$. Otro estudio presenta un porcentaje similar de personal capacitado sobre bioseguridad, mostrando además un nivel de conocimiento semejante ${ }^{(10)}$. Dos investigaciones presentaron personal con un nivel de conocimiento de bioseguridad bueno, tras haber sido capacitados. Esto no fue encontrado en nuestro estudio $^{(15,16)}$. Estas diferencias y semejanzas con otros estudios similares pueden estar expresando la necesidad de una capacitación sobre las medidas de bioseguridad más eficiente, además de dedicarle especial atención al personal de mayor edad y tiempo laborando en la institución.

En conclusión, el personal profesional del hospital no presenta un nivel de conocimiento ideal sobre las medidas de bioseguridad. Esto posiblemente conlleva a una deficiente situación de bioseguridad en el hospital, tanto para el personal profesional como técnico y pacientes.

\section{RECOMENDACIONES}

Actualizar al personal profesional en general sobre las medidas de bioseguridad, prestando especial atención al personal de enfermería, mayor de 60 años y con más de 3 años laborando en el hospital. Además de monitorizar el cumplimiento de dichas medidas. Es probable que el personal de salud técnico también lo necesite, aunque esto no haya sido evaluado en el presente estudio.

\section{REFERENCIAS BIBLIOGRÁFICAS}

1. Delgado M, Bedoya C, Robles L. Manual de Bioseguridad; Programa Nacional de Hemoterapia y Bancos de Sangre. LimaPerú: 2004.

2. Grupo de Trabajo del OMS,INFOSAN,FAO. Bioseguridad; Enfoque integrado de la gestión del riesgo para la vida y la salud de las personas, los animales y las plantas. Ginebra: 2010.

3. Albinagorta J, Tello J, Burga M, Roncal S, Bellido E, Ramirez P, et al. Manual de Salud Ocupacional. Oficina de Epidemiologia y Salud Ambiental. Lima: 2005

4. Garcia L, Facchino L. Occupational Accidents Among Brazilian Primary Health Care Workers: Sex and Occupation Inequalities, Institute for Applied Economic Research, Federal University of Pelotas CPMs Sessions. Brasilia: 2009; 1-14

5. Dirección General de Salud Ambiental, Ministerio de Salud. Plan Nacional de Prevención del VHB, VIH y la TB por Riesgo Ocupacional en los Trabajadores de Salud 2010-2015. Lima: 2010.

6. Ortega S, Martínez S, Pérez S, Fernández V, Bajac M, García L. Manual y Normas de Bioseguridad. UNNE [Internet]. 2012 [citado 12 de enero del 2012]: 3-5. Disponible en: http:/ / odn. unne.edu.ar/manbio.pdf

7. Wilburn S, Eijkemans G. La prevención de pinchazos con agujas en el personal de salud. Int J Occup Environ Health. 2004; 10:451-6

8. WHo. Seguridad del Personal de Salud. Ginebra: 2007

9. Raosoft. Sample Size Calculator. 2011. Disponible en: http://www.raosoft.com/samplesize.html

10. Iglesias M, Verdera J, Scull G, Arias M. Comportamiento de la Bioseguridad en un Área de Salud. Medimay. 2009; 15(1): 73-85

11. Soto V, Olano E. Conocimiento y cumplimiento de medidas de bioseguridad en personal de enfermería. Hospital Nacional Almanzor Aguinaga. Chiclayo 2002. Anales de la Facultad de Medicina Universidad Nacional Mayor de San Marcos. 2004; 65(2): 103-10.

12. Lubo A, Flores M, Quevedo A, Montiel M, Sirit Y, Petit M. Conocimiento y aplicación de las normas de bioseguridad por el personal de enfermería de una unidad de cuidados intensivos. Kasmera. 2004:32(2);71-9

13. Cuyubamba N. Conocimientos y Actitudes del Personal de Salud, hacia la Aplicación de las Medidas de Bioseguridad del Hospital Feliz Mayorca Soto Tarma-2003. [tesis doctoral]. Lima: Universidad Nacional de San Marcos; 2004

14. Vij A, Williamson A, Gupta S. Knowledge and Practice of a Nursing Staff Towards Infection Control Measures in a Tertiary Care Hospital. Journal of the Academy of Hospital. [Internet] 2001 [citado 21 de febrero del 2012];13(2): Disponible en: http://www.indmedica.com/journals. php?journalid=6\&issueid=20\&articleid=167\&action=article

15. Hernández E, Acosta M, Nadal B, Pijuan M, Fon Y, Armas N. Intervención educativa para incrementar los conocimientos sobre bioseguridad en el personal de enfermería de una institución hospitalaria. Rev Cubana Enfermer [Internet]. 2006 [citado 19 de febrero del 2012] ; 22(2). Disponible en: http://scielo.sld.cu/scielo.php?script=sci_ arttext\&pid=S0864-03192006000200008\&lng=es

16. Rodríguez O, Aguilera A, Barbé A, Delgado N. Intervención educativa sobre bioseguridad en trabajadores de la Salud. AMC [Internet]. 2010- [citado 19 de febrero del 2012]; 14(4): Disponible en: http://scielo.sld.cu/scielo.php?script=sci_ arttext\&pid=S1025-02552010000400012\&lng=es

\section{Fuentes de financiamiento:}

Este artículo ha sido financiado por el autor.

Conflicto de interés:

El autor declara no tener ningún conflicto de interés

\section{Correspondencia:}

Jorge A. Ruiz de Somocurcio-Bertocchi

Dirección: Calle Farallón Lt.10 Mz.E La Molina- Lima, Perú

Teléfono: 993925090

Correo electrónico: jorge_ruiz_de_somocurcio@hotmail.com

(c) La revista. Publicado por Universidad de San Martín de Porres, Perú. (сc) ву Licencia de Creative Commons Artículo en acceso abierto bajo términos de Licencia Creative Commons Atribución 4.0 Internacional. (http://creativecommons.org/licenses/by/4.0/) 